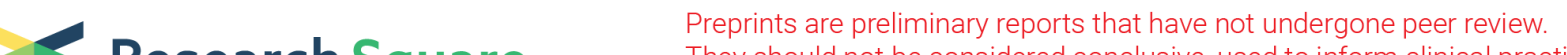 $\begin{array}{ll}\text { Research Square } & \text { They should not be considered conclusive, used to inform clinical practice, } \\ \text { or referenced by the media as validated information. }\end{array}$
}

\section{Evaluation of Matrix Metalloproteinase-7 and Matrix Metalloproteinase-9 as Biomarkers in Ureteropelvic Junction Obstruction in Children- a preliminary report}

\section{Chong Xie}

Xi'an international medical center hospital https://orcid.org/0000-0002-6726-6570

Xuan Liu

People's Hospital of xinjiang uygur Autonomous Region

Shuangli Qin

People's Hospital of xinjiang Uygur Autonomous Region

\section{Ling Zhou}

People's Hospital of xinjiang Uygur Autonomous Region

Liang Yu

People's Hospital of xinjiang Uygur Autonomous Regoin

Yanfang Liu

People's Hospital of xinjiang Uygur Autonomous Region

Shuixue Li ( $D$ 1298147216@qq.com )

Research article

Keywords: Ureteropelvic Junction Obstruction, Matrix Metalloproteinase-7, Matrix Metalloproteinase-9, Biomarkers

Posted Date: May 19th, 2020

DOI: https://doi.org/10.21203/rs.3.rs-27608/v1

License: (c) (i) This work is licensed under a Creative Commons Attribution 4.0 International License. Read Full License 


\section{Abstract \\ Background}

In children with congenital ureteropelvic junction obstruction (UPJO), Urinary biomarker may provide understanding of the health of the kidney, the need to intervene surgically, and the expected response and recoverability of the kidney following successful surgery. We evaluate whether the urinary matrix metalloproteinase-7(MMP-7) and matrix metalloproteinase-9(MMP-9) might be served as biomarkers in UPJO patients.

\section{Methods}

In this study, 24 unilateral UPJO patients and 16 healthy childrens were enrolled. Of which, 20 patients underwent ${ }^{99 \mathrm{~m}}$ Tc-DTPA (Diethylenetriaminepentaacetic Acid) renal dynamic imaging. And 16 patients underwent pyeloplasty and 8 others were followed. Postoperative follow-ups included ultrasonography, as well as MMP-7 and MMP-9 levels in urine, six months and one year after pyeloplasty. Study population was divided in 4 categories according to Society of Fetal Urology classification. For DTPA we subdivided the UPJO patients in 2 groups, obstructive (T 1/2 more than $20 \mathrm{~min}$ ) and partial obstructive (T 1/2 less than $20 \mathrm{~min}$ ). Quantitative urine protein analysis was performed and urinary creatinine levels were used to normalize protein levels for the markers analyzed.

\section{Results}

The results showed Creatinine-normalized urinary levels of MMP-7 and MMP-9 proteins were higher in patients with UPJO compared to healthy control subjects(Pख0.05). Moreover, urinary levels of MMP-7 and MMP-9 proteins in operation group were higher than non-operation group. Studies revealed that normalized urinary levels of MMP-7 and MMP-9 proteins in obstruction group were higher than partial obstruction group, although this differences were not statistically significant(P凶0.05). No significant differences in the Creatinine-normalized urinary levels of MMP-7 and MMP-9 in different degrees of hydronephrosis (P凶0.05). And normalized MMP-7 and MMP-9 levels were not correlated with the glomerular filtration rate of affected side $(r=-0.128, P=0.592 ; r=-0.157, P=0.507)$.

\section{Conclusions}

Compared to control group, the levels of MMP-7 and MMP-9 in urine of unilateral UPJO patients are significantly elevated, and they can potentially be used as determining indices for surgical plan. Although preliminary results indicate no significant correlation between them and clinical parameters, they would be potential biomarkers for UPJO. 


\section{Background}

Ureteropelvic junction obstruction (UPJO) is considered to be a form of upper urinary tract obstruction, with physiologic or structural etiologies, which leads to hydronephrosis of the affected kidney and also is the main cause of congenital obstructive nephropathy. UPJO has a very different clinical picture, which can vary from being asymptomatic to presenting with signs and symptoms like recurrent urinary tract infections, pain and hematuria. According to prenatal screening ultrasonography, hydronephrosis is the most common prenatal urological anomaly with a prevalence of $0.5 \%$ in newborns. However, UPJO only accounts for $35 \%$ of prenatal hydronephrotic cases ${ }^{[1]}$. Some patients with UPJO resolve spontaneously, while others require surgical intervention. Indications for surgical intervention include Worsening hydronephrosis or renal function, differential renal function less than $40 \%$, grade III or IV dilatation, anterior-posterior(AP) diameter of renal pelvic greater than $30 \mathrm{~mm}$, AP diameter of renal pelvic greater than $20 \mathrm{~mm}$ with calyceal dilation, and failure of conservative management ${ }^{[2,3]}$. Nonetheless, the optimal surveillance protocol is still under debate, the concern is both overtreatment and delayed treatment leading to impaired kidney function ${ }^{[1]}$. Imaging tests including ultrasound, MRU (magnetic resonance urography) and CTU (computed tomography urography) do not accurately reflect kidney function, and radionuclide renal scans is not generally available, especially in remote areas.

Therefore, it is necessary to find a more convenient and accurate predictors to evaluate renal function and degree of renal fibrosis. Urinary biomarker may provide understanding of the health of the kidney, the need to intervene surgically to alleviate obstruction, and the expected response and recoverability of the kidney following successful surgery ${ }^{[4]}$. Several urinary biomarkers have been evaluated in children with UPJO, including TGFB1, MCP1, LCN2, and IL $6^{[5-10]}$. However, the performance of these urinary biomarkers has been inconsistent in different studies, and it remains unclear whether this variation is due to biomarker performance, the patient's age, the grade of hydronephrosis, the degree of obstruction, the glomerular filtration rate(GFR), or whether repeated infection or not. Recent studies have shown that in adult patients with chronic kidney disease, the levels of matrix metalloproteinase-7 (MMP-7) and matrix metalloproteinase-9 (MMP-9) in urine are positively correlated with the degree of renal fibrosis and renal dysfunction, and the level of MMP-7 in urine is significantly increased when the renal function is slightly impaired without obvious fibrosis ${ }^{[11-13]}$. Therefore, we speculate that the level of MMP-7 and MMP-9 in urine may serve as non-invasive biomarkers for predicting clinical progression in patients with UPJO.

\section{Methods}

\section{Patients enrollment}

This study included patients who were seen at the Department of Pediatric Surgery, People's Hospital of Xinjiang Uygur Autonomous Region in China from January 2019 to February 2020. All urine samples came from the bladder. For bilateral UPJO, we were unable to group by some clinical parameters, such as grade of hydronephrosis, GFR and T1/2 of the affected kidney. Hence, our study included only unilateral 
UPJO patients. After obtaining institutional review board approval and parental consent, 24 patients, including 10 patients with UPJO on the left and 6 patients with UPJO on the right, were recruited to the study (Table 1). Most of the patients had no clear history of infection at first diagnosis. Of which, 4 patients had abnormal white blood cells in their urine at first diagnosis, and 5 patients had abnormal red blood cells in their urine at first diagnosis. Healthy control group consisted of 6 female and 10 male children those referred to a general pediatric outpatient clinic, where all were periodically monitored for their development and growth. All 16 children had no dilatation of renal pelvis(AP $₫ 5 \mathrm{~mm}$ ) and ureter by ultrasound, and no history of kidney disease.

Table 1

Comparison of general data between case group and control group

\begin{tabular}{|c|c|c|c|c|c|}
\hline & & Case group $(n=24)$ & Control group $(n=16)$ & $t / 2 / z$ & $P$ \\
\hline \multirow[t]{4}{*}{$\operatorname{Age}(y)$} & $<1$ & 11 & 3 & \multirow[t]{4}{*}{-1.509} & \multirow[t]{4}{*}{0.131} \\
\hline & $1 \sim 5$ & 6 & 5 & & \\
\hline & $6 \sim 10$ & 2 & 4 & & \\
\hline & $11 \sim 15$ & 5 & 4 & & \\
\hline \multirow[t]{2}{*}{ Gender } & Male & 20 & 10 & \multirow[t]{2}{*}{1.250} & \multirow[t]{2}{*}{0.264} \\
\hline & Female & 4 & 6 & & \\
\hline \multicolumn{2}{|c|}{ Urinary creatinine (umol/L) } & $4720.78 \pm 5237.22$ & $6099.25 \pm 6423.78$ & -0.751 & 0.461 \\
\hline \multicolumn{2}{|c|}{ MMP-7(ng/mL) } & $17.07 \pm 4.46$ & $8.37 \pm 2.13$ & 7.250 & $<0.001$ \\
\hline \multicolumn{2}{|c|}{ MMP-9(ng/mL) } & $43.70 \pm 9.61$ & $20.35 \pm 6.72$ & 8.431 & $<0.001$ \\
\hline
\end{tabular}

All patients were evaluated regarding AP diameter and cortical thickness of affected kidney by ultrasonography, which were all done by the same radiologist. Voiding cystourethrogram were performed to exclude vesicoureteral reflux. Of which, 20 patients underwent ${ }^{99 m}$ Tc-DTPA (Diethylenetriaminepentaacetic Acid) renal dynamic imaging. According to indications for surgical intervention mentioned above, 16 patients underwent pyeloplasty and 8 others were followed. Postoperative follow-ups included ultrasonography, as well as MMP-7 and MMP-9 levels in urine, six months and one year after pyeloplasty. So far, of the 16 patients undergoing surgery, 6 have had surgery for 6 months, but 3 have lost follow-up.

\section{Urine Samples Collection And Analysis}

Urine samples were obtained from the bladders of both UPJO patients and healthy controls. Fresh first morning voided urine specimens were centrifuged and stored within $6 \mathrm{~h}$ of collection at $-80^{\circ} \mathrm{C}$ until further analysis. Quantitative urine protein analysis was performed using commercially available human MMP7 
and MMP9 sandwich enzyme-linked immunosorbent assay (ELISA) kits (Enzyme-linked Biotechnology Co, Shanghai). Each specimen was analyzed in duplicate, and the concentration of the patient samples was determined by using standard curve analysis on generating a four-parameter logistic curve-fit. Because the random urine protein concentration is affected by a variety of factors, so urinary creatinine levels were used to normalize protein levels for the markers analyzed.

\section{Statistical analysis}

Data analysis was performed by SPSS24.0 software, and mapped by GraphPad Prism5. Statistical analysis was performed using the Kolmogorov-Smirnov test, $t$ test, ANOVA, Wilcoxon rank sum test, chisquare test and Spearman correlation analysis. The differences were considered statistically significant if $p<0.05$ for two-sided tests.

\section{Results}

\section{Comparison of general data between case group and control group}

The results showed that the difference of MMP-7 and MMP-9 between the case group and the control group was statistically significant (Pख0.05). There was no significant difference among other indicators (Pख0.05) (Table 1).

\section{Normality Test}

The normal test results of 40 samples showed that MMP-7 and MMP-9 were normally distributed, and Creatinine-normalized urinary MMP-9 levels (MMP9/ urine creatinine) was approximately skewed distribution(Table 2).

Table 2

Kolmogorov-Smirnov test

\begin{tabular}{|lllll|}
\hline & MMP-7 & Normalized MMP-7(\%o) & MMP-9 & Normalized MMP-9(\%) \\
\hline Mean & 13.588 & 7.668 & 34.362 & 19.345 \\
\hline Std. Deviation & 5.666 & 9.127 & 14.355 & 22.499 \\
\hline Kolmogorov-Smirnov Z & 0.680 & 1.662 & 0.705 & 1.316 \\
\hline Asymp. sig(2-tailed) & 0.744 & 0.008 & 0.703 & 0.063 \\
\hline
\end{tabular}




\section{Comparison of Normalized MMP7 and MMP9 levels between case group and control group}

The results showed Creatinine-normalized urinary levels of MMP-7 and MMP-9 proteins were higher in patients with UPJO compared to control subjects(Table 3).

Table 3

Comparison of Normalized MMP7 and MMP9 levels between case group and control group $[\mathrm{M}(25 \%, 75 \%)]$

\begin{tabular}{|lllll|}
\hline & Case group $(\mathrm{n}=24)$ & Control group $(\mathrm{n}=16)$ & $\mathrm{Z}$ & $\boldsymbol{P}$ \\
\hline Normalized MMP-7(\%) & $6.12[2.64,10.27]$ & $3.11[0.84,7.85]$ & -1.998 & 0.047 \\
\hline Normalized MMP-9(\%) & $15.97[6.58,15.97]$ & $6.78[1.90,20.82]$ & -2.153 & 0.031 \\
\hline
\end{tabular}

\section{Comparison of Normalized MMP7 and MMP9 levels between operation group and non-operaion group}

The patients were divided into two groups according to whether the operation was performed or not. The results showed Creatinine-normalized urinary levels of MMP-7 and MMP-9 proteins in operation group were higher than non-operation group (Table 4).

Table 4

Comparison of Normalized MMP7 and MMP9 levels between operation group and non- operation group $[\mathrm{M}(25 \%, 75 \%)]$

\begin{tabular}{|lll|}
\hline & Normalized MMP-7(\%o) & Normalized MMP-9(\%o) \\
\hline operation group $(\mathrm{n}=16)$ & $8.67[5.96,27.42]$ & $25.60[15.33,61.40]$ \\
\hline Non-operation group $(\mathrm{n}=8)$ & $3.10[1.29,5.27]$ & $8.95[3.39,15.13]$ \\
\hline Z & 3.166 & 2.922 \\
\hline P & 0.006 & 0.009 \\
\hline
\end{tabular}

\section{Comparison of Normalized MMP7 and MMP9 levels between obstruction group and partial obstruction group}

20 patients underwent ${ }^{99 \mathrm{~m}}$ Tc-DTPA renal dynamic imaging. For DTPA we subdivided the patients in 2 groups, obstruction group(T 1/2 more than $20 \mathrm{~min}$ ) and partial obstruction group(T 1/2 less than $20 \mathrm{~min}$ ). Our studies revealed that Creatinine-normalized urinary levels of MMP-7 and MMP-9 proteins in obstruction group were higher than partial obstruction group, although this differences were not statistically significant (Table 5 ). 
Table 5

Comparison of Normalized MMP7 and MMP9 levels between obstruction group and partial obstruction group $[\mathrm{M}(25 \%, 75 \%)]$

\begin{tabular}{|lll|}
\hline & Normalized MMP-7(\%o) & Normalized MMP-9(\%o) \\
\hline obstruction group $(\mathrm{n}=17)$ & $7.35[3.47,15.09]$ & $16.18[9.41,45.53]$ \\
\hline Partial obstruction group $(\mathrm{n}=3)$ & $2.46[2.32,]^{\star}$ & $5.56[5.39,]^{\star}$ \\
\hline Z & -1.641 & -0.081 \\
\hline P & 0.118 & 0.094 \\
\hline * The sample size was small, and the 75th percentiles could not be calculated. \\
\hline
\end{tabular}

\section{Comparison of Normalized MMP7 and MMP9 levels in different degrees of hydronephrosis}

24 UPJO patients were divided in 4 categories according to Society of Fetal Urology classification ${ }^{[14]}$. The research suggested that no significant differences in the Creatinine-normalized urinary levels of MMP-7 and MMP-9 in different degrees of hydronephrosis (Table 6).

Table 6

Comparison of Normalized MMP7 and MMP9 levels in different degrees of hydronephrosis $[\mathrm{M}(25 \%, 75 \%)]$

\begin{tabular}{|lll|}
\hline & \multicolumn{1}{l}{ Normalized MMP-7(\%o) } & Normalized MMP-9(\%) \\
\hline Grade $I(\mathrm{n}=2)$ & $4.68[2.32,]^{*}$ & $15.74[6.39,-]^{*}$ \\
\hline Grade $I I(\mathrm{n}=8)$ & $4.47[1.32,22.47]$ & $12.35[3.39,52.11]$ \\
\hline Grade III $(\mathrm{n}=7)$ & $7.37[3.20,10.36]$ & $16.18[7.14,31.06]$ \\
\hline Grade IV $(\mathrm{n}=7)$ & $7.35[5.80,29.96]$ & $22.06[14.32,61.86]$ \\
\hline $\mathrm{Z}$ & 2.013 & 1.707 \\
\hline$P$ & 0.570 & 0.638 \\
\hline * The sample size was small, and the 75th percentiles could not be calculated. \\
\hline
\end{tabular}

\section{Correlation analysis of Normalized MMP7 level, Normalized MMP9 level and affected side GFR}

The GFR of the affected kidney was determined by ${ }^{99 m}$ Tc-DTPA renal dynamic imaging analyzing. The result indicated that normalized MMP-7 levels were significantly correlated with normalized MMP-9 levels 
$(r=0.963, P<0.001)$. Normalized MMP-7 levels were not correlated with $G F R(r=-0.128, P=0.592)$, and Normalized MMP-9 levels also not correlated with GFR( $r=-0.157, P=0.507)($ Fig. 1$)$.

\section{Discussion}

The MMP-7 and MMP-9 are important members of the matrix metalloproteinases (MMPs). MMP-7 is one of the smallest MMPs, and is a secreted zinc- and calcium-dependent endopeptidase involved in regulating a diverse array of cellular processes, such as matrix remodeling, cell apoptosis, epithelialmesenchymal transition, and activating other MMPs members such as MMP-2, MMP-9 ${ }^{[15]}$. MMP-7 protein is almost undetectable in normal human kidneys ${ }^{[11]}$; however, its expression is markedly up-regulated in many types of adult chronic kidney diseases(CKDs), including IgA nephropathy, membranous nephritis, thrombotic microangiopathy, diabetic nephropathy, obstructive nephropathy, and chronic allograft nephropathy ${ }^{[16,17,11]}$.

MMP-7 protein is predominantly localized in renal tubular epithelium and glomerular podocytes of the diseased kidneys, and can be easily excreted into urine. Studies demonstrated that it not only can serve as a noninvasive biomarker for evaluating the degree of kidney fibrosis and renal dysfunction, but also is a pathologic mediator and potential therapeutic target of kidney fibrosis ${ }^{[11]}$.

Animal experiments showed that MMP-9 knockout mice reduce renal interstitial fibrosis in obstructive nephropathy ${ }^{[12]}$, furthermore deletion of MMP-7 did not affect renal expression of MMP-9 after unilateral ureteral obstruction ${ }^{[11]}$. Preliminary results showed the expression levels of MMP-9 in ureteropelvic junction tissue specimens was significantly higher in UPJO patients who were successfully treated by surgery compared to those did not have success, but these was no significant correlation between the expression levels of MMP-9 and obstructive DTPA, or grade of UPJO ${ }^{[18]}$.

Our results suggest that normalized urinary levels of MMP-7 and MMP-9 proteins were significantly higher in patients with unilateral UPJO compared to control subjects, and normalized MMP-7 levels were positively correlated with normalized MMP-9 level. Therefore, theoretically this conclusion should also apply to bilateral UPJO. Urinary levels of MMP-7 and MMP-9 in operation group were also significant higher than non-operation group. Consequently, they can potentially be used as determining indices for surgical plan. But to achieve this goal, further follow-up of patients who were not surgical candidate, needs to be performed.

Moreover, normalized urinary levels of MMP-7 and MMP-9 in obstruction group were higher than partial obstruction group, although the differences were not statistically significant. We hypothesized that obstruction resulted in a concentration of urine in the renal pelvis of the affected side, which indirectly led to an increase in the level of markers in the bladder.

However, normalized MMP-7 and MMP-9 levels were not correlated with the GFR of affected side. This result is inconsistent with earlier observation that the levels of urinary MMP-7 were closely correlated with 
the severity of kidney dysfunction ${ }^{[11]}$. There could be following reasons. Firstly, renal function is assessed in different ways. The GFR of the affected kidney was determined by ${ }^{99 \mathrm{~m}}$ Tc-DTPA renal dynamic imaging analyzing in our research. It may be affected by several factors, of which should be the quality of imaging agent injection and the choice of the background region of interest $(\mathrm{ROI})$, and the $\mathrm{ROI}$ of radionuclide retention and detention areas outlined deduction, which affect GFR value apparently ${ }^{[19,20]}$; and eGFR was used to represent the kidney function in previous research ${ }^{[11]}$. Secondly, in contrast to the random urine biomarker concentrations used in the above study ${ }^{[11]}$, urinary creatinine levels were used to normalize protein levels for the markers analyzed. Since creatinine excretion is relatively constant throughout the day and there is little individual difference, it can well reflect the excretion of 24-hour urine protein. And lastly, the participants in our study were unilateral UPJO patients, and the samples were from bladder urine. Therefore, the level of biomarkers in samples may be affected by a number of factors, such as the GFR of the affected kidney, the GFR of the contralateral healthy kidney and degree of obstruction. But in the study mentioned above, the study population was patients with CKDs which mostly involve bilateral kidneys and were not obstructive nephropathy ${ }^{[11]}$. Consequently, the level of biomarkers in bladder urine can reflect the impairment of renal function.

Our studies also revealed that no significant differences in the normalized MMP-7 and MMP-9 levels in different grades of hydronephrosis. Biomarkers in this study is measured in urine from bladder, which is noninvasive compared to detecting renal pelvic urine, although the latter may be more sensitive. As already noted, the level of biomarkers in bladder urine may be inevitably influenced by multiple factors, such as the grade of hydronephrosis, age, degree of obstruction, presence or absence of infection ${ }^{[21]}$, the GFR of the affected kidney and the contralateral healthy kidney. The diagnosis of UPJO is not difficult, and the ideal biomarker should be able to predict the clinical course of the disease, and provide the basis for surveillance.

Due to limited time and some patients lost follow-up, we were unable to conduct statistical analysis the changes of urinary MMP-7 and MMP-9 levels during the 6 months follow-up. Hence, the role of MMP-7 and MMP-9 in postoperative follow-up is unclear, Longer follow-up and more patients after surgery are necessary.

\section{Conclusion}

Compared to control group, the levels of MMP-7 and MMP-9 in urine of unilateral UPJO patients are significantly elevated, and they can potentially be used as determining indices for surgical plan.

Although preliminary results indicate no significant correlation between them and clinical parameters, they would be potential biomarkers for UPJO. Our results was from a small sample size in a single center. More and larger-scale studies are warranted.

\section{Abbreviations}


UPJO, Ureteropelvic junction obstruction; AP, anterior-posterior; MMP-7, matrix metalloproteinase-7; MMP9, matrix metalloproteinase-9; GFR, glomerular filtration rate; MRU, magnetic resonance urography; CTU, computed tomography urography; DTPA, Diethylenetriaminepentaacetic Acid; ELISA, enzyme-linked immunosorbent assay; ROI, background region of interest; MMPs, matrix metalloproteinases; CKDs, chronic kidney diseases.

\section{Declarations}

\section{Availability of data and materials}

Samples and data used in this study are in our Laboratory of People's Hospital of Xinjiang Uygur Autonomous Region, Urumqi, China

\section{Competing interests}

The authors declare that they have no competing interests.

\section{Consent for publication}

Not applicable.

\section{Ethics approval and consent to participate}

Subjects in both groups provided informed consent to participate the study and allowed their biological samples to be analyzed. Approval for the study was given by the ethical committee of People's Hospital of Xinjiang Uygur Autonomous Region (2018.10.19.KY89). Consent was signed by parent or responsible, once the patients were under 18 years.

\section{Funding}

This project was financially supported by Natural Science Foundation of XinJiang Uygur Autonomous Region. Grant No 2019D01C112.

\section{Authors' contributions}

Conception and design: CX, SXL; acquisition of patients and data: XL, SLQ, LZ, YFL; Drafting of the manuscript: CX, XL; enzyme-linked immunosorbent assay: LY; Administration support: SXL, LZ; Statistical 
Analysis: CX, XL; critical revision and important intellectual content: SXL; supervision: CX, SXL. We confirm that all authors read and aproved the final manuscript.

\section{Acknowledgment}

Not applicable.

\section{References}

1. Madsen MG, Nørregaard R, Frøkiær J. Jørgensen TM(2011)Urinary biomarkers in prenatally diagnosed unilateral hydronephrosis. Journal of Pediatric Urology7 (2):105-112. https://doi.org/10.1016/j.jpurol.2010.12.004.

2. Cangh PJV. Is it always necessary to treat a ureteropelvic junction syndrome? Current Urology Reports. 2007;8(2):118-21. https://doi.org/10.1007/s11934-007-0060-2.

3. 10.1007/s00467-007-0542-y

Yiee J, Wilcox D. (2008) Management of fetal hydronephrosis. Mayo Clinic Proceedings 23 (3):347353 https://doi.org/10.1007/s00467-007-0542-y.

4. $10.1007 / \mathrm{s} 00467-003-1074-8$

Chevalier RL, Peters CA. (2003)Congenital urinary tract obstruction: Proceedings of the State-Of-TheArt Strategic Planning Workshop-National Institutes of Health, Bethesda, Maryland, USA, 11-12 March 2002. Pediatric Nephorlogy18 (6):576-606 https://doi.org/10.1007/s00467-003-1074-8.

5. Merrikhi A, Bahraminia E. Association of urinary transforming growth factor- $\beta 1$ with the ureteropelvic junction obstruction. Adv Biomed Res. 2014;3:123. https://doi.org/10.4103/2277-9175.133196.

6. Sager C, Lopez JC, Duran V, Burek C, Perazzo E. Transforming growth factor- $\beta 1$ in congenital ureteropelvic junction obstruction: Diagnosis and follow-up. Int Braz J Urol. 2009;35(3):315-23. https://doi.org/10.1590/S1677-55382009000300008.

7. Karakuş S, Oktar T, Kucukgergin C, Kalelioglu I, Ziylan O. Urinary IP-10, MCP-1, NGAL, Cystatin-C and KIM-1 Levels in Prenatally-Diagnosed Unilateral Hydronephrosis: the Search for an Ideal Biomarker. Urology. 2015;87(10):185-92. https://doi.org/10.1016/j.urology.2015.09.007.

8. Madsen MG, Nørregaard R, Palmfeldt J, Olsen LH, Frøkiræ Jr, Jørgensen TM. Epidermal growth factor and monocyte chemotactic peptide-1: Potential biomarkers of urinary tract obstruction in children with hydronephrosis. Journal of Pediatric Urology. 2013;9(6):838-45. https://doi.org/10.1016/j.jpurol.2012.11.011.

9. Gerber C, Harel M, Lynch ML, Herbst KW, Shapiro LH. (2015) Proximal tubule proteins are significantly elevated in bladder urine of patients with ureteropelvic junction obstruction and may represent novel biomarkers: A pilot study. Journal of Pediatric Urology 12 (2):120.e121-120.e127 https://doi.org/10.1016/j.jpurol.2015.10.008. 
10. Wu B, Gong X, Kennedy WA, Brooks JD. Identification of transcripts associated with renal damage due to ureteral obstruction as candidate urinary biomarkers. American Journal of Physiology Renal Physiology. 2018;315(1):16-26. https://doi.org/10.1152/ajprenal.00382.2017.

11. Zhou D, Tian Y, L Sun L, Zhou L, Xiao L, Tan RJ, Tian J, Fu H, Hou FF, Liu Y. Matrix Metalloproteinase7 Is a Urinary Biomarker and Pathogenic Mediator of Kidney Fibrosis. J Am Soc Nephrol. 2017;28(2):598-611. https://doi.org/10.1681/ASN.2016030354.

12. Wang $X$, Zhou $Y$, Tan $R$, Xiong M, He W, Fang L, Wen P, Jiang L, Yang J. Mice lacking the matrix metalloproteinase-9 gene reduce renal interstitial fibrosis in obstructive nephropathy. Am J Physiol Renal Physiol. 2010;299(5):973-82. https://doi.org/10.1152/ajprenal.00216.2010.

13. Tan TK, Zheng GP, Hsu TT, Wang Y, Lee VW, Tian XR, Wang YP, Cao Q, Wang Y, Harris DC. Macrophage matrix metalloproteinase-9 mediates epithelial-mesenchymal transition in vitro in murine renal tubular cells. Am J Pathol. 2010;176(3):1256-70. https://doi.org/10.2353/ajpath.2010.090188.

14. Fernbach SK, Maizels M, Conway JJ. Ultrasound grading of hydronephrosis: Introduction to the system used by the Society for Fetal Urology. Pediatr Radiol. 1993;23(6):478-80. https://doi.org/10.1007/BF02012459.

15. Tan RJ, Liu Y. Matrix metalloproteinases in kidney homeostasis and diseases. American Journal of Physiology Renal Physiology. 2012;302(11):1351-61. https://doi.org/10.1152/ajprenal.00037.2012.

16. He WC, Tan RJ, Li YJ, Wang D, Nie J, Hou FF, Liu YH. Matrix Metalloproteinase-7 as a Surrogate Marker Predicts Renal Wnt/B-Catenin Activity in CKD. J Am Soc Nephrol. 2012;23(2):294-304. https://doi.org/10.1681/ASN.2011050490.

17. Surendran K, Simon TC, Liapis H, Mcguire JK. Matrilysin (MMP-7) expression in renal tubular damage: Association with Wnt4. Kidney Int. 2004;65(6):2212-22. https://doi.org/10.1111/j.15231755.2004.00641.x.

18. Reis ST, Leite KRM, Viana NI, Lopes RI, Moura CM, Ivanovic RF, Machado M, Denes FT, Giron A, Nahas. WC(2016)MMP9 overexpression is associated with good surgical outcome in children with UPJO: Preliminary results. BMC Urology 16 (1):44 https://doi.org/10.1186/s12894-016-0162-6.

19. Song C, Park H, Park S, Moon KH, Kim. KS(2007)The change in renal function in the supranormal hydronephrotic kidney after pyeloplasty. BJU International 99 (6):1483-1486 https://doi.org /10.1111/j.1464-410X.2007.06774.x.

20. Khan J, Charron M, Hickeson MP, Accorsi R, Qureshi S, Canning D(2004)Supranormal Renal Function in Unilateral Hydronephrotic Kidney Can Be Avoided. Clin Nucl Med 29 (7):410-4 https://doi.org /10.1097/01.rlu.0000129118.91958.16.

21. Grandaliano G, Gesualdo L, Bartoli F, Ranieri E, Monno R, Leggio A, Paradies G, Caldarulo E, Infante B, Schena FP. (2000) MCP-1 and EGF renal expression and urine excretion in human congenital obstructive nephropathy. Kidney Int 58 (1):182-92 https://doi.org /10.1046/j.15231755.2000.00153.x. 
Figures
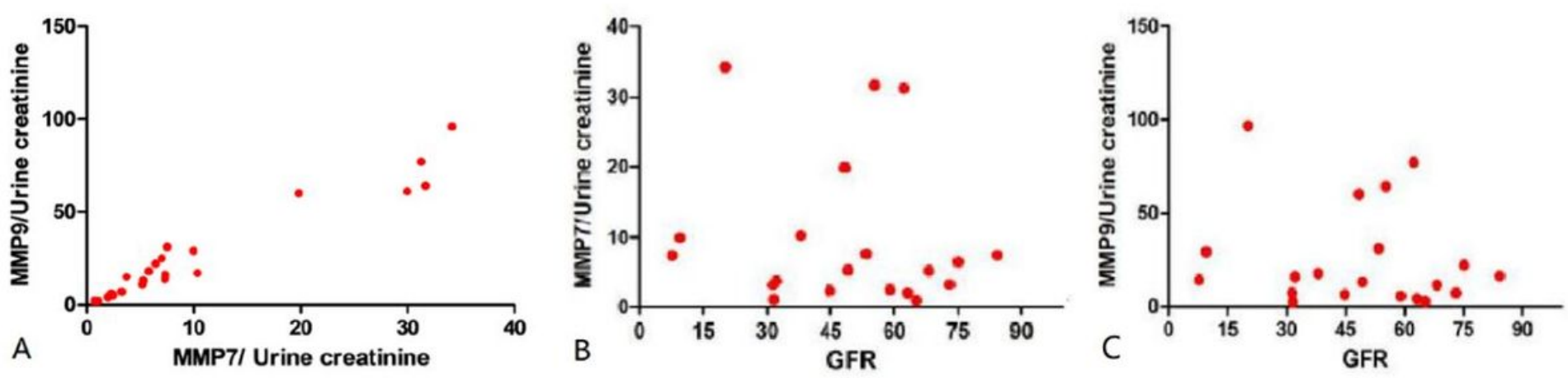

\section{Figure 1}

Correlation scatterplot. (A) Each point in the graph represents urinary MMP-7 and MMP-9 value in individual patients with UPJO (B) Each point in the graph represents urinary MMP-7 and GFR value in individual patients with UPJO (C) Each point in the graph represents urinary MMP-9 and GFR value in individual patients with UPJO

\section{Supplementary Files}

This is a list of supplementary files associated with this preprint. Click to download.

- originaldata.xlsx 